\title{
Pemetrexed-Erlotinib, Pemetrexed Alone, or Erlotinib Alone as Second-Line Treatment for East Asian and Non-East Asian Never-Smokers with Locally Advanced or Metastatic Nonsquamous Non-small Cell Lung Cancer: Exploratory Subgroup Analysis of a Phase II Trial
}

\author{
Dae Ho Lee, MD ${ }^{1}$ \\ Jung Shin Lee, MD' \\ Jie Wang, $M D^{2}$ \\ Te-Chun Hsia, MD \\ Xin Wang, $\mathrm{PhD}^{4}$ \\ Jongseok Kim, MD \\ Mauro Orlando, MD, $\mathrm{PhD}^{6}$
}

${ }^{1}$ Department of Oncology,

Asan Medical Center, Seoul, Korea,

${ }^{2}$ Department of Thoracic Medical Oncology,

Beijing Tumor Hospital and Institute,

Beijing, China,

${ }^{3}$ Department of Respiratory Therapy,

China Medical University and

China Medical University Hospital,

Taichung, Taiwan,

${ }^{4}$ Asia Pacific Statistical Sciences,

Lilly China Drug Development and

Medical Affairs Centre, Shanghai, China,

${ }^{5}$ Medical Department, Lilly Korea Ltd.,

Seoul, Korea,

${ }^{6}$ Medical Department, Eli-Lilly Interamerica Inc.,

Buenos Aires, Argentina

\begin{abstract}
Purpose
This subgroup analysis of a phase II trial was conducted to assess possible ethnicity-based trends in efficacy and safety in East Asian (EA) and non-EA populations with nonsquamous non-small cell lung cancer (NSCLC).
\end{abstract}

\begin{abstract}
Materials and Methods
Never-smoker patients $(n=240)$ with locally advanced or metastatic nonsquamous NSCLC included $133 \mathrm{EA}$ patients randomized to pemetrexed supplemented with dexamethasone, folic acid, and vitamin $B_{12}$ plus erlotinib (pemetrexed-erlotinib) $(n=41)$, erlotinib $(n=49)$, or pemetrexed $(n=43)$, and 107 non-EA patients randomized to pemetrexed-erlotinib $(n=37)$, erlotinib $(n=33)$, or pemetrexed $(n=37)$. The primary endpoint, progression-free survival (PFS), was analyzed using a multivariate Cox model.
\end{abstract}

\section{Results}

Consistent with the results of the overall study, a statistically significant difference in PFS among the three arms was noted in the EA population favoring pemetrexed-erlotinib (overall $p=0.003$ ) as compared with either single-agent arm (hazard ratio [HR], 0.48; 95\% confidence interval [Cl], 0.29 to 0.79 ; $\mathrm{p}=0.004$ vs. erlotinib; $\mathrm{HR}, 0.40 ; 95 \% \mathrm{Cl}, 0.23$ to 0.70 ; $p=0.001$ vs. pemetrexed). The EA patients treated with pemetrexed-erlotinib achieved a longer median PFS (7.4 months) compared with erlotinib (4.5 months) and pemetrexed (4.0 months). The PFS results also numerically favored pemetrexed-erlotinib in the non-EA population (overall $\mathrm{p}=0.210)(\mathrm{HR}, 0.62 ; 95 \% \mathrm{Cl}, 0.37$ to $1.05 ; \mathrm{p}=0.078$ vs. erlotinib; $\mathrm{HR}$, $0.75 ; 95 \% \mathrm{Cl}, 0.42$ to $1.32 ; \mathrm{p}=0.320$ vs. pemetrexed) (median PFS: pemetrexed-erlotinib, 6.7 months; erlotinib, 3.0 months; pemetrexed, 4.4 months).

\section{Conclusion}

The PFS results from this subset analysis in both EA and non-EA populations are consistent with the results in the overall population. The PFS advantage for pemetrexed-erlotinib is significant compared with the single agents in EA patients.

Correspondence: Mauro Orlando, MD, PhD Eli Lilly Interamerica, Tronador 4890, Piso 12, Argentina

Tel: 54-11-45464053

Fax: 54-11-45464171

E-mail: mauro@lilly.com

Received February 25, 2014

Accepted May 25, 2014

Published online November 24, 2014

\section{Key words}

Nonsquamous non-small cell lung cancer, East Asian,

Pemetrexed, Erlotinib

\section{Introduction}

Despite standard platinum-based chemotherapy and newer targeted therapies, patients with advanced non-small cell lung cancer (NSCLC) inevitably experience disease progression. In addition to the use of maintenance and sec- ond-line therapies as standard-of-care, there is an increasing need for the appropriate use of targeted and tailored therapies to improve efficacy and tolerability in patients with advanced NSCLC.

Pemetrexed (Alimta, Eli Lilly and Company), a multitargeted antifolate, is a standard-of-care for first-line [1], maintenance [2], and second-line [3] treatment of nonsquamous 
advanced NSCLC. Furthermore, a subgroup analysis of a phase III global study showed that pemetrexed plus platinum therapy improved overall survival (OS) compared with gemcitabine plus platinum therapy in a subgroup of East Asian (EA) patients with advanced nonsquamous NSCLC [4]. Results from Asian studies have also demonstrated the efficacy of pemetrexed in the second-line setting $[5,6]$.

Erlotinib (Tarceva, OSI-774, OSI Pharmaceuticals / Genentech), a reversible, epidermal growth factor receptor (EGFR) tyrosine kinase inhibitor (TKI) as monotherapy has previously demonstrated significantly improved survival versus placebo in patients with advanced NSCLC as a second- or third-line therapy $[7,8]$. Activating mutations in the EGFR gene are associated with a high sensitivity to EGFR TKIs and the treatment of patients with NSCLC carrying EGFRmutations with EGFR TKIs results in prolongation of progression-free survival (PFS) and higher response rates compared with standard chemotherapy [9]. In addition, it has been reported that EGFR mutations are present predominantly in women never-smokers, individuals with adenocarcinoma and those of EA ethnicity [10-12].

Previous attempts to combine chemotherapy and EGFR TKIs have produced mixed results in unselected populations [13-16]. Currently, there is no consensus on the use and additive effect of chemotherapy and TKIs in the treatment of advanced NSCLC. In an attempt to improve the outcomes of the combination therapy, Lee et al. [17] conducted a study comparing erlotinib plus pemetrexed versus each single agent in a clinically enriched population of never-smoker patients with advanced nonsquamous NSCLC. In this study, pemetrexed-erlotinib combination therapy significantly improved PFS compared with either drug alone in this selected population. Upon subsequent testing, the incidence of EGFR mutations in this clinically selected population who provided evaluable samples was $55.8 \%$.

In an exploratory analysis of patients with EGFR mutations, the combination therapy showed a PFS advantage over pemetrexed but not over erlotinib; OS was numerically improved for the combination over both single agents [17]. Due to the distinctive characteristics of EGFR-expressing NSCLC in Asian patients compared with Caucasian patients, this exploratory analysis assessed the possible association of ethnicity (EA and non-EA populations) with efficacy and safety in the phase II global trial [17].

\section{Materials and Methods}

\section{Study design and treatment plan}

Details regarding the study design and treatment were described previously [17]. This was an exploratory analysis of the multicenter, open-label, randomized, phase II global trial conducted in never-smokers with advanced nonsquamous NSCLC by Lee et al. [17]. In this trial, 247 patients who had histological or cytological diagnosis of locally advanced or metastatic NSCLC, had failed only one prior chemotherapy regimen, and were eligible for further chemotherapy following disease progression were randomly assigned in a ratio of 1:1:1 to pemetrexed plus erlotinib, erlotinib alone, or pemetrexed alone. Patients with squamous histology $(n=7)$ were excluded from the primary analysis. In the exploratory analysis reported here, only those patients enrolled in China, Hong Kong, Korea, and Taiwan were considered for inclusion in the EA population $(\mathrm{n}=133,55.4 \%)$ and patients enrolled in Australia, Brazil, India, and the United Kingdom were considered for inclusion in the non-EA population $(\mathrm{n}=107,44.6 \%)$.

Pemetrexed $\left(500 \mathrm{mg} / \mathrm{m}^{2}\right)$ was administered intravenously on day 1 of a 3-week cycle. Erlotinib (150 mg, orally) as a single agent was administered once daily continuously and, when taken in combination with pemetrexed, once daily from day 2 through day 14 of a 3-week cycle. All patients treated with pemetrexed received dexamethasone, folic acid, and vitamin $B_{12}$. Patients continued to receive study therapy until disease progression, or any other discontinuation criterion was met.

The global trial [17] was conducted according to the Declaration of Helsinki and good clinical practice guidelines and was approved by each participating institution and its ethical review board. All patients signed a written informed consent form before treatment. The ClinicalTrials.gov Identifier is NCT00550173.

\section{Baseline and treatment assessments}

Details of the treatment assessments have been previously reported [17]. Tumors were measured by the same imaging method(s) within 4 weeks before randomization, every 6 weeks ( \pm 1 week) during therapy, and every 6 weeks $( \pm 1$ week) post-study-drug therapy, until disease progression, death, or 18 months after randomization for patients with objective response (complete response or partial response) or stable disease at the time of discontinuation of study therapy. Details of the biomarker analysis have been previously reported [17]. 


\section{Statistical analyses}

The primary efficacy endpoint in the global trial was PFS and secondary endpoints included OS, objective response rate (ORR), and safety assessment [17]. In this report, PFS, OS, ORR, and toxicities between treatment arms in the EA and non-EA populations were reported. The analyses in the EA and non-EA populations used the same methodology as that used in the primary analysis of the qualified intentionto-treat (ITT) population [17]. The reverse Kaplan-Meier estimator methodology [18] was used to calculate the median follow-up time.

Post-hoc exploratory subgroup analyses for PFS and OS were conducted on selected subgroups of EA and non-EA patients using the same multivariate Cox regression models adjusted for Eastern Cooperative Oncology Group performance status (ECOG PS; 0-1 vs. 2), sex (male vs. female), and age ( $\leq 65$ years vs. $>65$ years). All tests were conducted at a two-sided 0.05 significance level unless otherwise stated and $\mathrm{p}$-values were not adjusted for multiplicity. The incidence of EGFR mutations was calculated as the number of EGFR mutations over the number of EGFR evaluable samples. Due to the small sample size of the EGFR subgroups (mutationpositive and mutation-negative) in each treatment arm, comparative analyses between arms were not appropriate. Instead, descriptive analyses of PFS and OS were conducted on EGFR subgroups.

Safety endpoints were analyzed on nonsquamous patients who received at least one dose of study therapy and according to the treatment they received in the first cycle. Safety analyses included summaries of the incidence of adverse events (AEs) by maximum Common Terminology Criteria for Adverse Events (CTCAE) ver. 3.0 grade.

\section{Results}

\section{Patients and treatment}

The 240 patients comprised 133 EA patients randomized to erlotinib-pemetrexed $(n=41)$, erlotinib $(n=49)$, or pemetrexed $(n=43)$, and 107 non-EA patients randomized to erlotinib-pemetrexed $(n=37)$, erlotinib $(n=33)$, or pemetrexed $(\mathrm{n}=37)$. The proportion of female patients was comparatively higher than the proportion of male patients in the EA and non-EA populations (Table 1). The proportion of patients with PS 1 was higher than the proportion of patients with PS 0 in the EA and non-EA populations. A higher proportion of patients in the EA population were from the Republic of Korea $(39.8 \%$, 53/133). Similarly, a higher proportion of patients in the non-EA population were from India $(62.6 \%$, $67 / 107)$. The EGFR mutation status was unknown in the majority of patients in both of these populations (EA, $102 / 133$ [76.7\%] and non-EA, 95/107 [88.8\%]). Within the limits of a relatively small number of samples tested, the proportion of patients with EGFR mutation was comparatively higher in the EA population with known test results $(61.3 \%, 19 / 31)$ compared with those in the non-EA population $(41.7 \%, 5 / 12)$. Notably, the EGFR mutation status was not known at baseline and therefore, it was not used as a stratification factor.

\section{Efficacy}

\section{1) Primary efficacy measure}

The analysis of the primary endpoint, PFS, was conducted for the EA and non-EA populations (Fig. $1 \mathrm{~A}$ and C). In the EA population, a statistically significant difference in PFS was observed among the three arms (overall $p=0.003$ ), with the pemetrexed-erlotinib combination significantly improving PFS as compared with either single-agent arm (pemetrexed-erlotinib vs. erlotinib, $\mathrm{p}=0.004$; pemetrexed-erlotinib vs. pemetrexed, $\mathrm{p}=0.001$ ) (Table 2 ). No statistically significant difference was noted between pemetrexed and erlotinib $(\mathrm{p}=0.430)$ (Table 2). The EA patients treated with the pemetrexed-erlotinib combination had a longer median PFS (7.4 months; 95\% CI, 5.3 to 15.3 months) compared with those treated with erlotinib (4.5 months; $95 \%$ CI, 2.7 to 11.1 months; $\mathrm{p}=0.016)$ and pemetrexed (4.0 months; 95\% CI, 2.9 to 6.7 months; $\mathrm{p}=0.006$ ).

In the non-EA population, the PFS results also numerically favored the pemetrexed-erlotinib combination; however, the PFS differences were not statistically significant (overall $\mathrm{p}=0.210$ ) for the pemetrexed-erlotinib combination compared with either agent alone (pemetrexed-erlotinib vs. erlotinib, $\mathrm{p}=0.078$; pemetrexed-erlotinib vs. pemetrexed, $\mathrm{p}=0.320$ ) (Table 2). Similarly, no statistically significant difference was noted between pemetrexed and erlotinib ( $\mathrm{p}=0.505)$ (Table 2). The median PFS in the pemetrexed-erlotinib arm was 6.7 months (95\% CI, 1.8 to 13.2 months) compared with 3.0 months for erlotinib (95\% CI, 1.8 to 5.6 months; $\mathrm{p}=0.061$ ) and 4.4 months for pemetrexed (95\% CI, 2.1 to 7.2 months; $\mathrm{p}=0.512$ ).

\section{2) Secondary efficacy measures}

In the EA population, numerically improved OS was noted among the three treatment arms (Fig. 1B), but the OS did not reach statistical significance (overall $\mathrm{p}=0.114$; pemetrexederlotinib vs. erlotinib: hazard ratio [HR], 0.83 ; and pemetrexed-erlotinib vs. pemetrexed: HR, 0.53) (Table 2). 
Table 1. Baseline demographic and disease characteristics (East Asian and non-East Asian ITT population)

\begin{tabular}{|c|c|c|c|c|c|c|}
\hline \multirow[b]{2}{*}{ Parameter } & \multicolumn{3}{|c|}{ East Asian } & \multicolumn{3}{|c|}{ Non-East Asian } \\
\hline & $\begin{array}{c}\text { Pemetrexed } \\
+ \text { erlotinib }(n=41)\end{array}$ & $\begin{array}{l}\text { Erlotinib } \\
\qquad(n=49)\end{array}$ & $\begin{array}{l}\text { Pemetrexed } \\
\qquad(n=43)\end{array}$ & $\begin{array}{c}\text { Pemetrexed } \\
+ \text { erlotinib }(n=37)\end{array}$ & $\begin{array}{l}\text { Erlotinib } \\
\qquad(n=33)\end{array}$ & $\begin{array}{l}\text { Pemetrexed } \\
(\mathrm{n}=37)\end{array}$ \\
\hline \multicolumn{7}{|l|}{ Gender } \\
\hline Male & $8(19.5)$ & $14(28.6)$ & $15(34.9)$ & $12(32.4)$ & $14(42.4)$ & $20(54.1)$ \\
\hline Female & $33(80.5)$ & $35(71.4)$ & $28(65.1)$ & $25(67.6)$ & $19(57.6)$ & $17(45.9)$ \\
\hline \multicolumn{7}{|l|}{ Age (yr) } \\
\hline Mean \pm SD & $57.0 \pm 11.30$ & $56.2 \pm 9.38$ & $54.8 \pm 12.60$ & $55.0 \pm 12.23$ & $50.5 \pm 11.25$ & $57.6 \pm 13.54$ \\
\hline Range & $33.5-72.5$ & $31.7-74.4$ & $26.2-73.6$ & $30.0-76.0$ & $32.0-74.7$ & $34.0-87.0$ \\
\hline \multicolumn{7}{|l|}{ Country } \\
\hline China & $14(34.1)$ & $17(34.7)$ & $15(34.9)$ & 0 & 0 & 0 \\
\hline Hong Kong & 0 & $3(6.1)$ & $2(4.7)$ & 0 & 0 & 0 \\
\hline Republic of Korea & $20(48.8)$ & $20(40.8)$ & $13(30.2)$ & 0 & 0 & 0 \\
\hline Taiwan, Province of China & $7(17.1)$ & 9 (18.4) & $13(30.2)$ & & & \\
\hline Australia & 0 & 0 & 0 & $3(8.1)$ & 0 & 0 \\
\hline Brazil & 0 & 0 & 0 & $15(40.5)$ & $5(15.2)$ & $8(21.6)$ \\
\hline India & 0 & 0 & 0 & $15(40.5)$ & $27(81.8)$ & $25(67.6)$ \\
\hline United Kingdom & 0 & 0 & 0 & $4(10.8)$ & $1(3.0)$ & $4(10.8)$ \\
\hline \multicolumn{7}{|l|}{ ECOG performance status } \\
\hline 0 & 0 & $8(16.3)$ & $7(16.3)$ & $10(27.0)$ & $12(36.4)$ & $9(24.3)$ \\
\hline 1 & $38(92.7)$ & $40(81.6)$ & $34(79.1)$ & $23(62.2)$ & $16(48.5)$ & $26(70.3)$ \\
\hline 2 & $3(7.3)$ & $1(2.0)$ & $2(4.7)$ & $3(8.1)$ & $5(15.2)$ & $2(5.4)$ \\
\hline 3 & 0 & 0 & 0 & $1(2.7)$ & 0 & 0 \\
\hline \multicolumn{7}{|l|}{ EGFR mutation status } \\
\hline Yes & $5(12.2)$ & $8(16.3)$ & $6(14.0)$ & $2(5.4)$ & 0 & $3(8.1)$ \\
\hline No & $5(12.2)$ & $4(8.2)$ & $3(7.0)$ & $5(13.5)$ & $2(6.1)$ & 0 \\
\hline Unknown & $31(75.6)$ & $37(75.5)$ & $34(79.1)$ & $30(81.1)$ & $31(93.9)$ & $34(91.9)$ \\
\hline \multicolumn{7}{|l|}{ Pathological diagnosis } \\
\hline Adenocarcinoma & $40(97.6)$ & $48(98.0)$ & $43(100)$ & $32(86.5)$ & $28(84.8)$ & $34(91.9)$ \\
\hline Mixed cell lung carcinoma & $1(2.4)$ & 0 & 0 & $1(2.7)$ & $1(3.0)$ & 0 \\
\hline Large cell lung carcinoma & 0 & $1(2.0)$ & 0 & $4(10.8)$ & $4(12.1)$ & $3(8.1)$ \\
\hline \multicolumn{7}{|l|}{ Stage of disease ${ }^{\text {a) }}$ at entry } \\
\hline IIIA & 0 & 0 & 0 & 0 & $2(6.1)$ & $1(2.7)$ \\
\hline IIIB & $3(7.3)$ & $8(16.3)$ & $7(16.3)$ & $3(8.1)$ & $4(12.1)$ & $4(10.8)$ \\
\hline IV & $38(92.7)$ & $41(83.7)$ & $36(83.7)$ & $34(91.9)$ & $27(81.8)$ & $32(86.5)$ \\
\hline
\end{tabular}

Values are presented as number (\%) unless otherwise indicated. ITT, intent-to-treat; SD, standard deviation; ECOG, Eastern Cooperative Oncology Group; EGFR, epidermal growth factor receptor. ${ }^{\text {a) }}$ Non-small cell lung cancer diagnosis stage IIIA, IIIB, or IV was per the definition by the American Joint Committee on Cancer staging criteria for lung cancer.

Similarly, in the non-EA population, the HR for PFS favored the combination (pemetrexed-erlotinib vs. erlotinib: HR, 0.62; pemetrexed-erlotinib vs. pemetrexed: HR, 0.75); however, in contrast to the HRs for OS in the EA population, the HRs for OS in the non-EA population did not show any clear advantage in favor of the combination (pemetrexederlotinib vs. erlotinib: HR, 1.42; pemetrexed-erlotinib vs. pemetrexed: HR, 1.00) (Table 2).

A long OS was noted in this second-line NSCLC population, independent of treatment arm [17]. This was particu- larly evident in the EA population, in which the median OS was 25.0 months (95\% CI, 20.1 to 37.5 months) for pemetrexed-erlotinib, 25.3 months (95\% CI, 18.2 to not applicable) for erlotinib, and 19.5 months (95\% CI, 11.8 to 26.9 months) for pemetrexed. Also in the non-EA population, longer than expected OS occurred (Fig. 1D), although the medians for OS in the non-EA population were shorter than those in the EA patients. The median OS was 19.2 months $(95 \%$ CI, 4.5 to 20.5 months) for pemetrexed-erlotinib, 20.6 months (95\% CI, 6.8 to 25.3 months) for erlotinib, and 12.7 months (95\% CI, 6.9 to 
Table 2. Cox regression analysis of PFS and OSa) adjusted by covariates ${ }^{\text {a) }}$ (East Asian and non-East Asian ITT population)

\begin{tabular}{|c|c|c|c|c|c|c|c|c|}
\hline \multirow{3}{*}{ Variable } & \multicolumn{4}{|c|}{ East Asian } & \multicolumn{4}{|c|}{ Non-East Asian } \\
\hline & \multicolumn{2}{|c|}{ PFS $^{\text {b) }}$} & \multicolumn{2}{|c|}{$\mathrm{OS}^{\mathrm{b})}$} & \multicolumn{2}{|c|}{ PFS $^{\text {b) }}$} & \multicolumn{2}{|c|}{$\mathrm{OS}^{\mathrm{b})}$} \\
\hline & HR $(95 \%$ CI) & p-value $e^{c)}$ & HR $(95 \%$ CI) & p-value $e^{c)}$ & HR $(95 \%$ CI $)$ & p-value ${ }^{c}$ & HR $(95 \%$ CI) & p-value ${ }^{c)}$ \\
\hline $\begin{array}{l}\text { Treatment adjusted } \\
\text { by covariates (overall) }\end{array}$ & & 0.003 & & 0.114 & & 0.210 & & 0.528 \\
\hline $\begin{array}{l}\text { Pemetrexed+erlotinib } \\
\text { vs. erlotinib } \\
\text { (reference level) }\end{array}$ & $\begin{array}{c}0.48 \\
(0.29-0.79)\end{array}$ & 0.004 & $\begin{array}{c}0.83 \\
(0.44-1.56)\end{array}$ & 0.554 & $\begin{array}{c}0.62 \\
(0.37-1.05)\end{array}$ & 0.078 & $\begin{array}{c}1.42 \\
(0.72-2.80)\end{array}$ & 0.306 \\
\hline $\begin{array}{l}\text { Pemetrexed+erlotinib } \\
\text { vs. pemetrexed } \\
\text { (reference level) }\end{array}$ & $\begin{array}{c}0.40 \\
(0.23-0.70)\end{array}$ & 0.001 & $\begin{array}{c}0.53 \\
(0.28-1.00)\end{array}$ & 0.051 & $\begin{array}{c}0.75 \\
(0.42-1.32)\end{array}$ & 0.320 & $\begin{array}{c}1.00 \\
(0.55-1.79)\end{array}$ & 0.993 \\
\hline $\begin{array}{l}\text { Pemetrexed } \\
\text { vs. erlotinib } \\
\text { (reference level) }\end{array}$ & $\begin{array}{c}1.20 \\
(0.76-1.90)\end{array}$ & 0.430 & $\begin{array}{c}1.56 \\
(0.88-2.75)\end{array}$ & 0.124 & $\begin{array}{c}0.83 \\
(0.48-1.43)\end{array}$ & 0.505 & $\begin{array}{c}1.43 \\
(0.72-2.83)\end{array}$ & 0.309 \\
\hline
\end{tabular}

PFS, progression-free survival; OS, overall survival; ITT, intent-to-treat; $\mathrm{HR}$, hazard ratio; $\mathrm{CI}$, confidence intervals. ${ }^{\text {a) }} \mathrm{PFS}$ and OS were adjusted by three covariates: performance status (0-1 vs. 2), histologic subtype (adenocarcinoma vs. non-adenocarcinoma), and sex (female vs. male), b)PFS was defined as the time from randomization to the first date of progressive disease (either objectively determined or clinical progression) or death from any cause; OS was defined as the time from randomization to the date of death from any cause; survival time was censored at the date of last contact for patients who were still alive or lost to follow-up, 'The p-value was based on the Wald's test, with a two-sided significance level of 0.05 .

23.1 months) for pemetrexed. In the non-EA population, no statistically significant difference in OS was observed among the three treatment arms (overall $\mathrm{p}=0.528$ ) (Table 2, Fig. 1). The median length of follow-up from the date of enrollment was 22.4 months (95\% CI, 20.1 to 25.6 months) in EA patients and 18.4 months (95\% CI, 12.3 to 29.6 months) in non-EA patients.

Consistent with the results of improved PFS for the pemetrexed-erlotinib combination, a statistically significant difference in ORR was observed among the three arms in the EA population $(p<0.001)$ (Table 3$)$. The ORR was $51.2 \%$ for pemetrexed-erlotinib, $30.6 \%$ for erlotinib, and $11.6 \%$ for pemetrexed. Overall, a statistically significant difference in ORR was observed across the three arms (overall $p<0.001$ ), with the pemetrexed-erlotinib combination resulting in significantly higher ORR compared with either single-agent arm (pemetrexed-erlotinib vs. erlotinib, $\mathrm{p}=0.027$; pemetrexed-erlotinib vs. pemetrexed, $p<0.001$ ) (Table 3). There was a numerical, but not statistically significant, difference in the disease control rate (DCR) among the three treatment arms $(\mathrm{p}=0.107)$. For pemetrexed-erlotinib, the DCR was 70.7\% (Table 3). Pemetrexed-erlotinib had a significantly higher DCR than erlotinib alone $(\mathrm{p}=0.033)$ or pemetrexed alone ( $\mathrm{p}=0.048$ ) (Table 3).

In the non-EA population, a statistically significant difference in ORR was noted among the three treatment arms $(\mathrm{p}=0.013)$ (Table 3). The ORR was $37.1 \%$ for pemetrexed- erlotinib, $27.3 \%$ for erlotinib, and $8.1 \%$ for pemetrexed. Pemetrexed-erlotinib had a significantly higher ORR than pemetrexed $(\mathrm{p}=0.010)$. The ORR for pemetrexed-erlotinib was not significantly higher than that for erlotinib $(\mathrm{p}=0.389)$ (Table 3$)$. No statistically significant difference in the DCR was noted among the three treatment arms ( $\mathrm{p}=0.804)$. The DCR was $57.1 \%$ for pemetrexed-erlotinib, $54.5 \%$ for erlotinib, and $62.2 \%$ for pemetrexed (Table 3 ).

\section{Exploratory analyses}

Exploratory subgroup analyses for the EA and non-EA populations are presented in Fig. 2A and B. Subgroup analyses of PFS in the EA population showed that the benefit of the combination was consistent across most clinical subsets including sex, ECOG PS, and age (Fig. 2A and B). In the non-EA population, the pemetrexed-erlotinib combination resulted in numerically higher PFS over pemetrexed alone or erlotinib alone for most subgroups (Fig. 2E and F). No statistically significant difference for OS was observed among treatment arms (Fig. 2C, D, G, and H). PFS and OS results for the ECOG PS 2 subset were not reported for the EA and non-EA populations due to the small sample sizes.

In the EA population, the EGFR mutation status (positive or negative) was available for 31 patients and the incidence of EGFR mutation was $61.2 \%$ (19/31 patients). In the non-EA population, EGFR mutation status was available for 12 
A

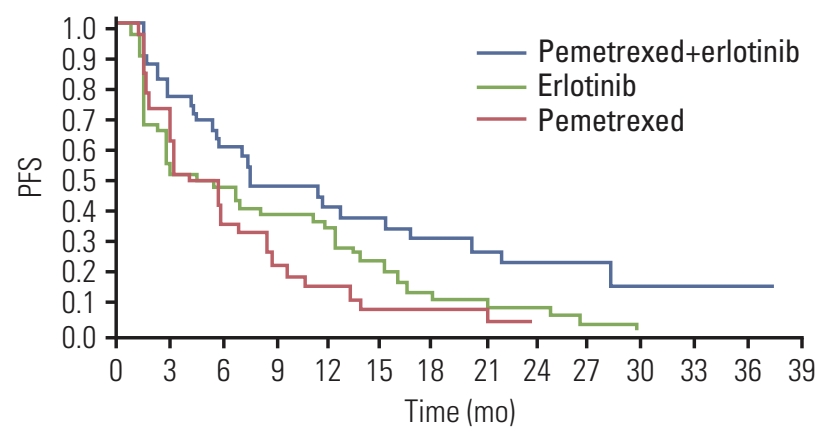

Patients at risk:

Pemetrexed+erlotinib

$\begin{array}{lllllllllll}41 & 28 & 22 & 15 & 12 & 11 & 9 & 7 & 5 & 5 & 2\end{array}$

Erlotinib

$\begin{array}{llllllllll}49 & 26 & 22 & 18 & 16 & 11 & 6 & 5 & 3 & 1\end{array}$

Pemetrexed

$\begin{array}{lllllllll}43 & 23 & 13 & 6 & 4 & 2 & 2 & 2 & 1\end{array}$

C

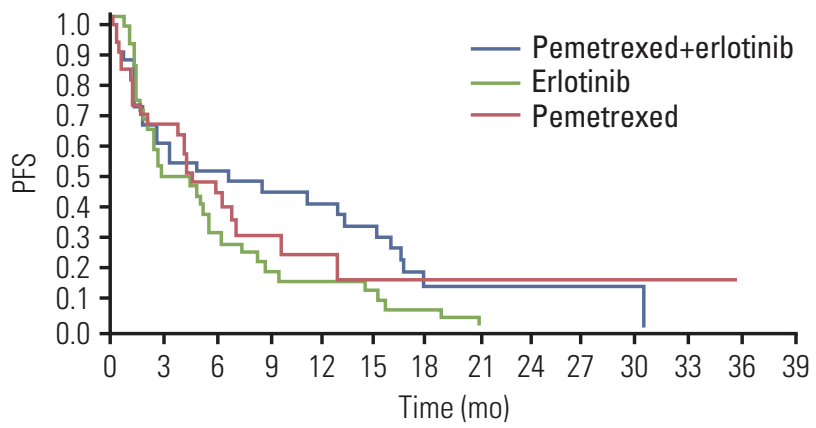

Patients at risk:

Pemetrexed+erlotinib

$\begin{array}{lllllllllll}37 & 20 & 16 & 12 & 11 & 9 & 3 & 3 & 3 & 3 & 3\end{array}$

Erlotinib

$\begin{array}{lllllll}33 & 16 & 10 & 6 & 5 & 4 & 2\end{array}$

Pemetrexed

$\begin{array}{llllll}37 & 21 & 15 & 6 & 4 & 2\end{array}$

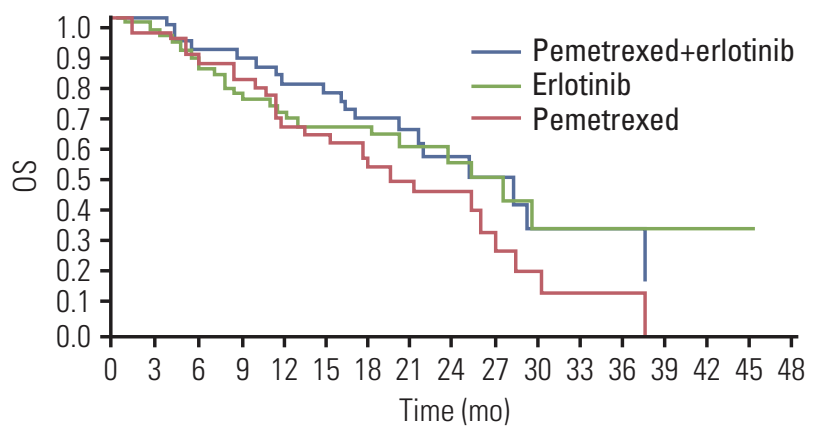

Patients at risk:

Pemetrexed+erlotinib

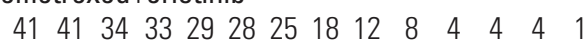

Erlotinib

$\begin{array}{lllllllllll}49 & 47 & 42 & 36 & 34 & 32 & 32 & 15 & 11 & 10 & 4\end{array}$

Pemetrexed

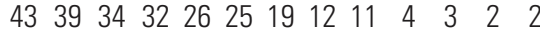

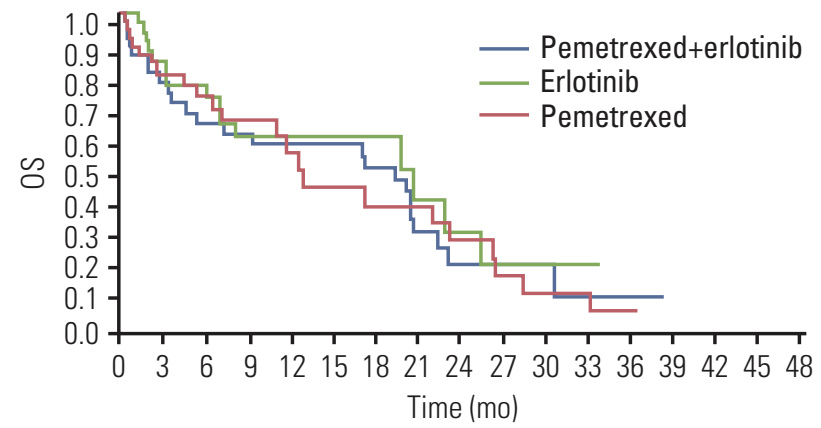

Patients at risk:

Pemetrexed+erlotinib

$\begin{array}{llllllllllll}37 & 25 & 20 & 19 & 18 & 18 & 14 & 7 & 4 & 4 & 4 & 1\end{array}$

Erlotinib

$33251815151515 \quad 4 \quad 3 \quad 2$

Pemetrexed

$\begin{array}{llllllllllll}37 & 25 & 21 & 17 & 11 & 8 & 7 & 7 & 5 & 3 & 2 & 1\end{array}$

Fig. 1. Kaplan-Meier plots of progression-free survival (PFS) and overall survival (OS) for East Asian (EA) and non-EA populations. (A) Kaplan-Meier plot of PFS for EA population. (B) Kaplan-Meier plot of OS for EA population. (C) Kaplan-Meier plot of PFS for non-EA population. (D) Kaplan-Meier plot of OS for non-EA population. For OS, 53.7\%, 37.2\% and $53.1 \%$ of EA patients and $35.1 \%, 40.5 \%$ and $54.5 \%$ of non-EA patients treated with pemetrexed-erlotinib, pemetrexed or erlotinib, respectively, were censored.

patients, and the incidence of EGFR mutation was $41.7 \%$ (5/12) (Table 1).

Descriptive comparisons of PFS and OS by EGFR mutation subsets for the EA and non-EA populations are presented in Appendix 1. In the overall and EA populations, EGFR mutation positive patients generally had longer PFS compared with EGFR negative patients, independent of treatment received (Appendix 1A-D). In the EA population, EGFR mutation-negative patients treated with erlotinib seemed to have shorter PFS compared with EA patients treated with pemetrexed alone or the pemetrexed-erlotinib combination (Appendix 1E). Among the three arms, the combination arm appeared to provide the best PFS benefit in EGFR mutationnegative patients in the EA population (Appendix 1E). OS was similar across treatment arms and EGFR mutation status subsets in the EA and non-EA populations, with few exceptions (Appendix $1 \mathrm{~F}$ and $\mathrm{H}$ ). The small sample size, however, limits the interpretation of the observed trends. 


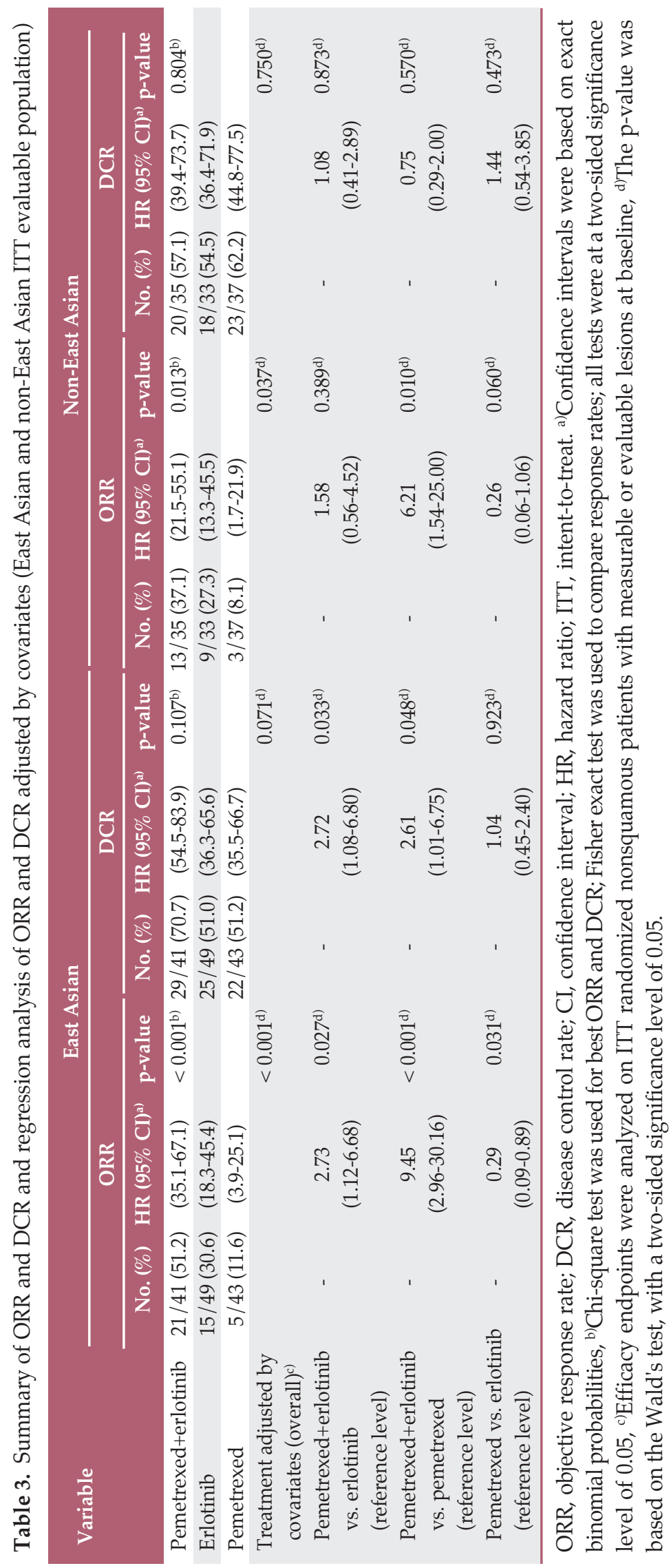


Table 4. Summary of study drug-related TEAEs by maximum CTCAE grade $\geq 3$ (East Asian and non-East Asian safety population $^{\text {a)})}$

\begin{tabular}{|c|c|c|c|c|c|c|}
\hline \multirow[b]{2}{*}{ Toxicity } & \multicolumn{3}{|c|}{ East Asian } & \multicolumn{3}{|c|}{ Non-East Asian } \\
\hline & $\begin{array}{c}\text { Pemetrexed } \\
\text { +erlotinib }(n=40)\end{array}$ & $\begin{array}{l}\text { Erlotinib } \\
\qquad(n=50)\end{array}$ & $\begin{array}{l}\text { Pemetrexed } \\
\quad(n=40)\end{array}$ & $\begin{array}{c}\text { Pemetrexed } \\
\text { +erlotinib }(n=35)\end{array}$ & $\begin{array}{l}\text { Erlotinib } \\
\quad(n=33)\end{array}$ & $\begin{array}{l}\text { Pemetrexed } \\
\qquad(n=36)\end{array}$ \\
\hline \multicolumn{7}{|l|}{ Non-laboratory parameters } \\
\hline \multicolumn{7}{|l|}{ Grade $3 / 4 \geq 5 \%$} \\
\hline Mucositis/stomatitis & $4^{\mathrm{b})}$ & 0 & 0 & 0 & 0 & 0 \\
\hline Rash: acne/acneiform & 3 & 4 & 0 & 1 & 1 & 0 \\
\hline Diarrhea & 2 & 0 & 0 & 5 & 0 & 0 \\
\hline Fatigue (asthenia, lethargy, ma & nalaise) 2 & 0 & 2 & 2 & 0 & 1 \\
\hline Rash: desquamation & 1 & 0 & 0 & 2 & 0 & 0 \\
\hline Anorexia & 1 & 0 & 0 & 0 & 0 & 2 \\
\hline \multicolumn{7}{|l|}{ Grade 5} \\
\hline Sudden death & 0 & 0 & 0 & 0 & 0 & 1 \\
\hline Viral hepatitis & 0 & 0 & 0 & 0 & 0 & 1 \\
\hline \multicolumn{7}{|l|}{ Laboratory parameters } \\
\hline \multicolumn{7}{|l|}{ Grade $3 / 4 \geq 5 \%$} \\
\hline Neutropenia & 11 & 0 & 5 & 7 & 0 & 5 \\
\hline Leukopenia & 3 & 0 & 3 & 6 & 0 & 3 \\
\hline Anemia & 4 & 0 & 3 & 4 & 0 & 4 \\
\hline Lymphopenia & 3 & 0 & 4 & 6 & 0 & 1 \\
\hline Hyperglycemia & 2 & 0 & 1 & 0 & 0 & 0 \\
\hline Thrombocytopenia & 0 & 0 & 1 & 2 & 0 & 1 \\
\hline Hyponatremia & 0 & 0 & 0 & 1 & 1 & 2 \\
\hline Hypokalemia & 0 & 0 & 0 & 1 & 0 & 2 \\
\hline \multicolumn{7}{|l|}{ Grade 5} \\
\hline Neutropenia & 0 & 0 & 0 & 0 & 0 & 1 \\
\hline Other blood/bone marrow & 0 & 0 & 0 & 2 & 0 & 0 \\
\hline
\end{tabular}

Values are presented as number (\%). TEAEs, treatment-emergent adverse events; CTCAE, Common Terminology Criteria for Adverse Events; NOS, not otherwise specified. a)Safety endpoints were analyzed on nonsquamous patients who received at

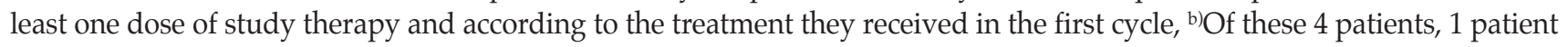
$(2.5 \%)$ experienced mucositis / stomatitis (clinical examination) and 3 patients $(7.5 \%)$ experienced mucositis / stomatitis (functional/symptomatic).

\section{Post-discontinuation treatment}

Overall, in the EA population, 90 patients $(67.7 \%)$ received at least one post-discontinuation treatment (PDT). In particular, 63 patients $(47.4 \%)$ received systemic chemotherapy, 36 $(27.1 \%)$ received EGFR TKIs, and 26 patients $(19.5 \%)$ received radiation therapy. In comparison, fewer patients $29(27.1 \%)$ in the non-EA population received at least one PDT (systemic chemotherapy in 19 patients [17.8\%]; EGFR TKIs in 7 patients [6.5\%]; and radiation therapy in 8 patients [7.5\%]).

\section{Safety}

The overall incidence of at least one high-grade (grade 3/4) study drug-related treatment-emergent adverse events
(TEAEs) was low in both the EA (39 [30.0\%]) and non-EA (38 [36.5\%]) populations. The pemetrexed-erlotinib combination in the EA population generally had a higher incidence of drug-related grade 3 or 4 TEAEs than either single agent alone (Table 4). Similarly, in the non-EA population, patients in the pemetrexed-erlotinib combination arm generally had a higher incidence of drug-related grade 3 or 4 TEAEs than either single agent alone. Common drug-related grade 3 and 4 TEAEs in pemetrexed-erlotinib, erlotinib, and pemetrexed in the EA population included neutropenia $(27.5 \%, 0 \%$, and $15.0 \%)$, anemia $(10.0 \%, 0 \%$, and $7.5 \%)$, and lymphopenia $(7.5 \%, 0 \%$, and $10.0 \%)$. Common drug-related grade 3 and 4 TEAEs in pemetrexed-erlotinib, erlotinib, and pemetrexed in the non-EA population included neutropenia $(20.0 \%, 0 \%$, and $13.9 \%)$, leukopenia $(17.1 \%, 0 \%$, and $8.3 \%)$, and lym- 
A

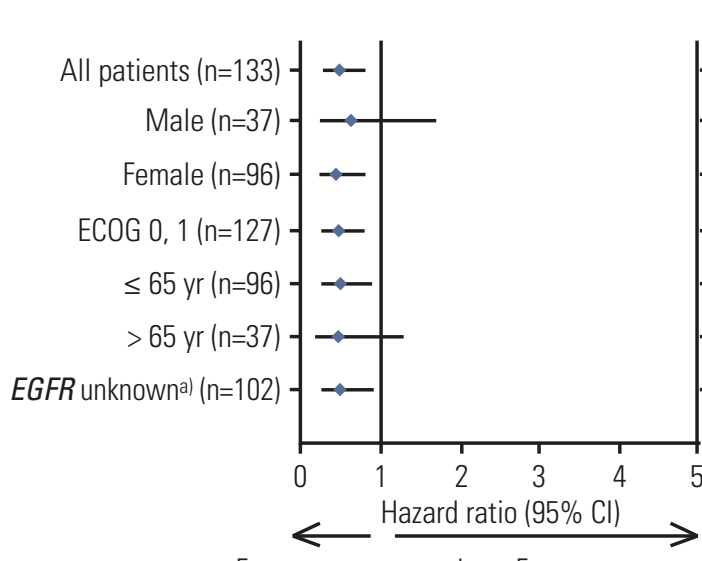

Favours pemetrexed Favours pemetrexed +erlotinib
$\operatorname{HR}(95 \% \mathrm{Cl})$

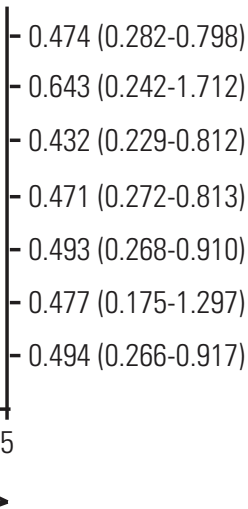

$-0.474(0.282-0.798)$
$-0.643(0.242-1.712)$
$-0.432(0.229-0.812)$
$-0.471(0.272-0.813)$
$-0.493(0.268-0.910)$
$-0.477(0.175-1.297)$
$-0.494(0.266-0.917)$
5$$
\text { Favours pemetrexed Favours erlotinib }
$$
terlotinib

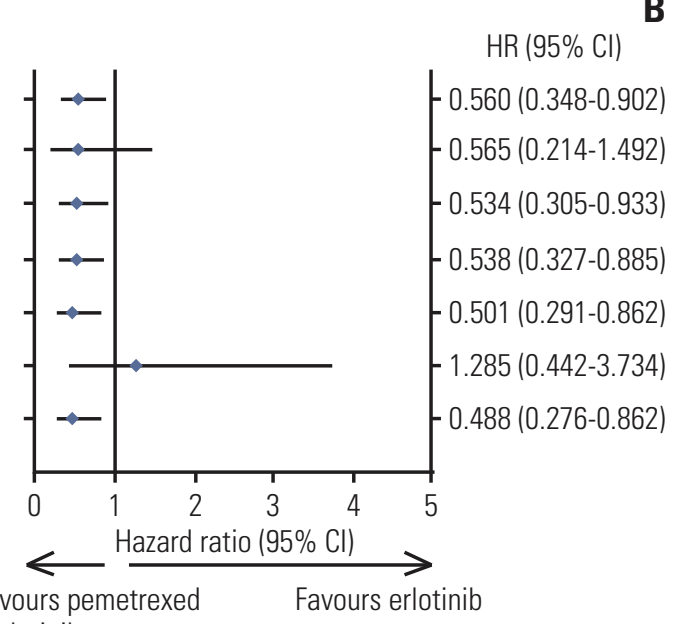

C

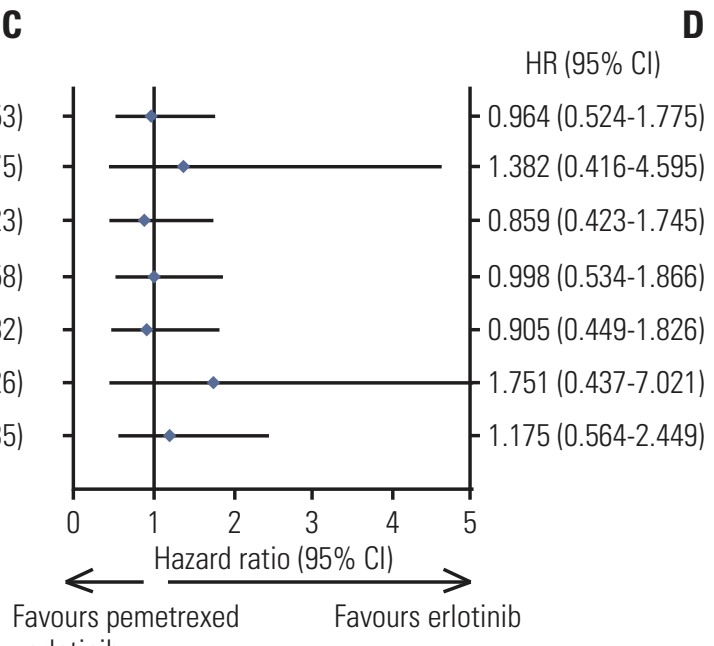

Fig. 2. Forest plots by clinical subgroups (qualified intention-to-treat population). (A, B) Forest plots of progression-free survival (PFS) for East Asian (EA) population by clinical subgroups. (C, D) Forest plots of overall survival (OS) for EA population by clinical subgroups. (Continued to the next page)

phopenia $(17.1 \% 0 \%$, and $2.8 \%$ ) (Table 4). No drug-related deaths were reported in the EA population. Five non-EA patients $(2$ in the pemetrexed-erlotinib arm and 3 in the pemetrexed arm) died during the study from study drugrelated AEs (Table 4).

\section{Discussion}

This is an exploratory subgroup analysis of the EA and
non-EA populations in the recently reported phase II, randomized, controlled trial [17]. In this trial, the combination of pemetrexed-erlotinib was compared with either pemetrexed or erlotinib alone as second-line treatment in a selected population of never-smoking patients with locally advanced or metastatic nonsquamous NSCLC [17].

In this global trial [17], never-smoker patients were selected in an attempt to "enrich" the patient population for potential EGFR TKI sensitivity. At the inception of this study, data from the Iressa Pan-Asia Study (IPASS) [19] trial and the critical importance of EGFR mutational status were not available to determine sensitivity to EFGR TKIs. Therefore, molecular profiling of patients for EGFR mutations was only 

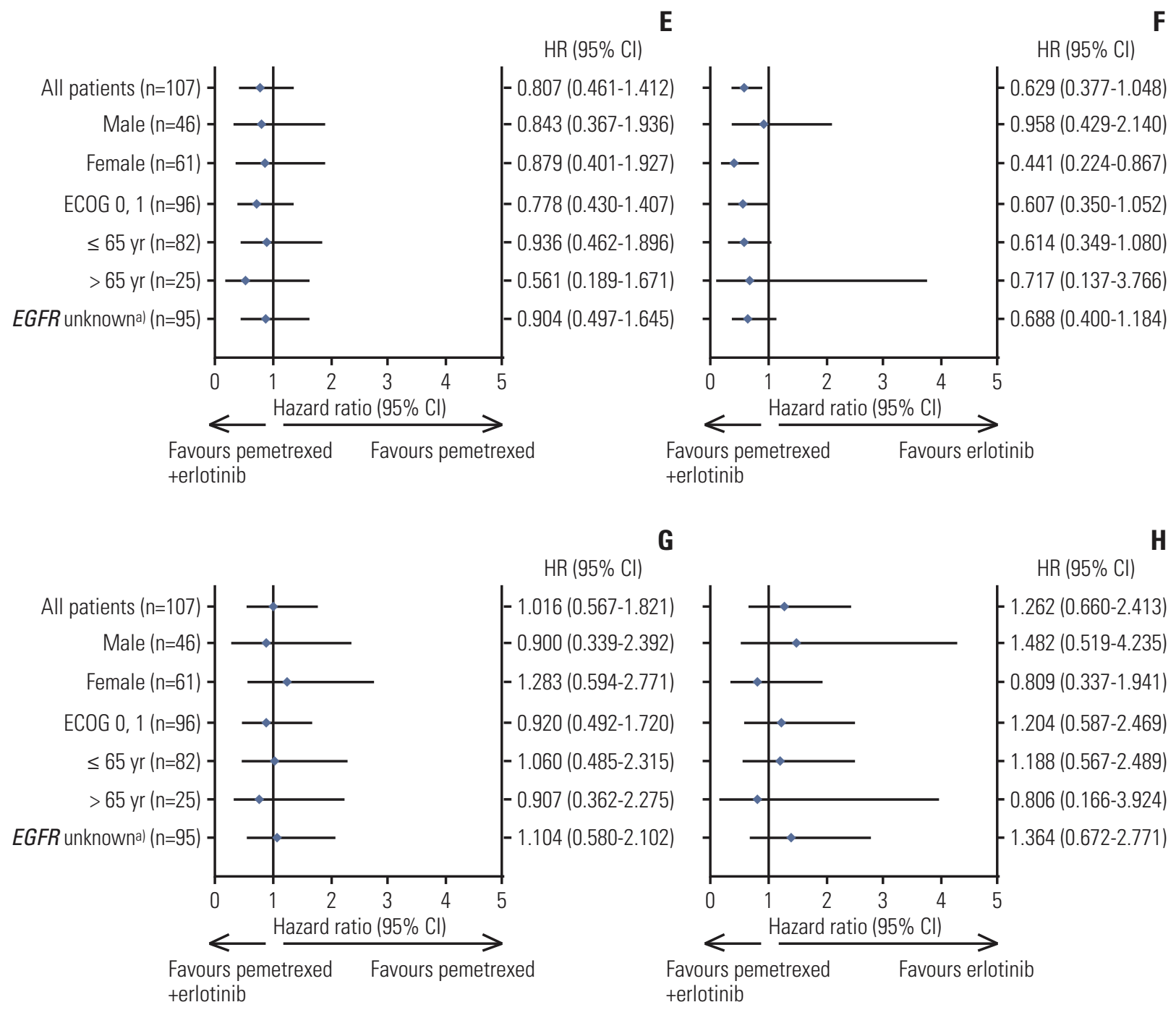

Fig. 2. (Continued from the previous page) (E, F) Forest plots of PFS for non-EA population by clinical subgroups. (G, H) Forest plots of OS for non-EA population by clinical subgroups. CI, confidence interval; EGFR, epidermal growth factor receptor; $\mathrm{HR}$, hazard ratio. a)Patient with any EGFR mutation detected was categorized as mutated (positive); patient without EGFR mutations detected was categorized as non-mutated (negative); patient without EGFR samples or without valid EGFR samples was categorized as unknown. Note: The results for Eastern Cooperative Oncology Group performance status (ECOG PS) 2 subgroup were removed from the plot due to the extremely small number of patients in the EA $(n=6)$ and non-EA $(\mathrm{n}=10)$ populations.

included as an exploratory analysis in this study. The results from the current subgroup analysis support other findings [20] that the pemetrexed-erlotinib combination enhances activity compared with single-agent treatment.

The PFS results in this exploratory subgroup analysis were consistent with the results of the PFS results in the overall qualified ITT population [17], particularly for the EA population, demonstrating that the pemetrexed-erlotinib combination significantly prolonged PFS and led to a longer median PFS compared with either agent alone. Similarly, the pemetrexed-erlotinib combination therapy had numerically superior PFS in the non-EA population; however, the difference in the non-EA population was not significant $(\mathrm{p}=0.210)$. Subgroup analyses of PFS in the EA population in this study also showed that the benefit of the combination was consistent across the clinical subsets including gender, ECOG PS, age, and EGFR-positive status. Note that the analysis was powered only for PFS in the overall ITT population, not any 
particular subgroup populations. It is expected that the statistical tests, when restricted to subgroup populations (e.g., non-EA), are underpowered. Thus, the results reported in this manuscript need to be interpreted in the context of this limitation.

Overall, numerically improved but not statistically significant OS was noted among the three treatment arms in the EA, non-EA, and ITT [17] populations. Of note, neither the global trial [17] nor this subgroup analysis was powered for OS. Similar to the ITT population [17], among the three treatment arms, the censoring rates were high in the EA and non-EA populations, which may also have limited the analysis for OS. In addition, the median OS in the EA population was notably long, ranging from 19.5 to 25.3 months among the three treatment arms and from 12.7 to 20.6 months in the non-EA population. The longer median OS in EA patients is consistent with longer median OS reported previously [21] and may be due to several factors, including the higher incidence of EGFR mutations and the more frequent use of PDT, including the addition of cross-over treatment with pemetrexed and EGFR TKIs. The pemetrexed-erlotinib combination also had a stronger effect on ORR and DCR in the EA population compared with that in the non-EA population.

Recently, increased evaluation of biomarkers has enhanced our understanding of the complex NSCLC biology and has aided in the development of targeted drugs and tailored strategies for the treatment of advanced NSCLC. In a prospective biomarker study in patients with NSCLC, EGFR mutation has been identified as the strongest predictive factor for outcomes with EGFR TKIs [22].

In our study, never-smoking status was used as a clinical enriching factor for sensitivity to EGFR TKIs, which led to a higher proportion of patients with EGFR mutations. As expected, the incidence of EGFR mutation in patients evaluated for a somatic mutation was higher in the EA and non-EA populations, at $61 \%$ and $42 \%$, respectively, than in the average Caucasian [23] or Asian populations with NSCLC. In comparison, the IPASS [19] and First-SIGNAL [24] studies reported $60 \%$ and $44 \%$ of EA never-smokers or former light smokers with pulmonary adenocarcinoma were positive for EGFR mutations, respectively. As a limitation of this study, the EGFR mutation status was unknown for the majority of patients (EA population, $76.7 \%$; vs. non-EA population, $88.8 \%$ ).

In this study, pemetrexed-erlotinib improved PFS compared with single agents alone in the EA population. The pemetrexed-erlotinib combination therapy had numerically superior PFS in the non-EA population. Given the small number of patients, the descriptive analysis of EGFR mutation status (positive and negative) in the EA and non-EA populations cannot conclusively identify clear trends. The fact that the majority of patients on this study had unknown EGFR mutation status greatly limits the conclusions and underscores the importance of routine molecular profiling in advanced NSCLC.

\section{Conclusion}

In conclusion, the subset analysis presented in this study and recent findings suggest that the combination of EGFR TKIs and pemetrexed may benefit selected populations of patients with nonsquamous NSCLC. This hypothesis needs to be tested in prospective randomized clinical trials of second- or first-line therapy in patients with EGFR profiling to expand our insight regarding the role of chemotherapy and EGFR TKI combinations.

\section{Conflicts of Interest}

Dae Ho Lee has received honoraria from Eli Lilly and Company as a member of advisory board. Xin Wang, Jongseok Kim, and Mauro Orlando are employees of Eli Lilly and Company, with some owning stock in Eli Lilly and Company. All other authors declare no conflicts of interest.

\section{Acknowledgments}

We thank all of the patients and investigators who participated in this study. We also thank Sharad Wankhade and Noelle Gasco of InVentiv Health Clinical for writing and editorial support, respectively. This study was sponsored by Eli Lilly and Company. 


\section{References}

1. Scagliotti GV, Parikh P, von Pawel J, Biesma B, Vansteenkiste J, Manegold C, et al. Phase III study comparing cisplatin plus gemcitabine with cisplatin plus pemetrexed in chemotherapynaive patients with advanced-stage non-small-cell lung cancer. J Clin Oncol. 2008;26:3543-51.

2. Ciuleanu T, Brodowicz T, Zielinski C, Kim JH, Krzakowski M, Laack E, et al. Maintenance pemetrexed plus best supportive care versus placebo plus best supportive care for non-smallcell lung cancer: a randomised, double-blind, phase 3 study. Lancet. 2009;374:1432-40.

3. Hanna N, Shepherd FA, Fossella FV, Pereira JR, De Marinis F, von Pawel J, et al. Randomized phase III trial of pemetrexed versus docetaxel in patients with non-small-cell lung cancer previously treated with chemotherapy. J Clin Oncol. 2004;22: 1589-97.

4. Yang CH, Simms L, Park K, Lee JS, Scagliotti G, Orlando M. Efficacy and safety of cisplatin/pemetrexed versus cisplatin/ gemcitabine as first-line treatment in East Asian patients with advanced non-small cell lung cancer: results of an exploratory subgroup analysis of a phase III trial. J Thorac Oncol. 2010;5: 688-95.

5. Sun Y, Wu YL, Zhou CC, Zhang L, Zhang L, Liu XY, et al. Second-line pemetrexed versus docetaxel in Chinese patients with locally advanced or metastatic non-small cell lung cancer: a randomized, open-label study. Lung Cancer. 2013;79:143-50.

6. Ohe Y, Ichinose Y, Nakagawa K, Tamura T, Kubota K, Yamamoto N, et al. Efficacy and safety of two doses of pemetrexed supplemented with folic acid and vitamin B12 in previously treated patients with non-small cell lung cancer. Clin Cancer Res. 2008;14:4206-12.

7. Shepherd FA, Rodrigues Pereira J, Ciuleanu T, Tan EH, Hirsh $\mathrm{V}$, Thongprasert $\mathrm{S}$, et al. Erlotinib in previously treated nonsmall-cell lung cancer. N Engl J Med. 2005;353:123-32.

8. Bezjak A, Tu D, Seymour L, Clark G, Trajkovic A, Zukin M, et al. Symptom improvement in lung cancer patients treated with erlotinib: quality of life analysis of the National Cancer Institute of Canada Clinical Trials Group Study BR.21. J Clin Oncol. 2006;24:3831-7.

9. Kaneda H, Yoshida T, Okamoto I. Molecularly targeted approaches herald a new era of non-small-cell lung cancer treatment. Cancer Manag Res. 2013;5:91-101.

10. Rosell R, Moran T, Queralt C, Porta R, Cardenal F, Camps C, et al. Screening for epidermal growth factor receptor mutations in lung cancer. N Engl J Med. 2009;361:958-67.

11. Kosaka T, Yatabe Y, Endoh H, Kuwano H, Takahashi T, Mitsudomi T. Mutations of the epidermal growth factor receptor gene in lung cancer: biological and clinical implications. Cancer Res. 2004;64:8919-23.

12. Shigematsu H, Lin L, Takahashi T, Nomura M, Suzuki M, Wistuba II, et al. Clinical and biological features associated with epidermal growth factor receptor gene mutations in lung cancers. J Natl Cancer Inst. 2005;97:339-46.

13. Giaccone G, Herbst RS, Manegold C, Scagliotti G, Rosell R,
Miller V, et al. Gefitinib in combination with gemcitabine and cisplatin in advanced non-small-cell lung cancer: a phase III trial--INTACT 1. J Clin Oncol. 2004;22:777-84.

14. Herbst RS, Giaccone G, Schiller JH, Natale RB, Miller V, Manegold $\mathrm{C}$, et al. Gefitinib in combination with paclitaxel and carboplatin in advanced non-small-cell lung cancer: a phase III trial: INTACT 2. J Clin Oncol. 2004;22:785-94.

15. Herbst RS, Prager D, Hermann R, Fehrenbacher L, Johnson BE, Sandler A, et al. TRIBUTE: a phase III trial of erlotinib hydrochloride (OSI-774) combined with carboplatin and paclitaxel chemotherapy in advanced non-small-cell lung cancer. J Clin Oncol. 2005;23:5892-9.

16. Gatzemeier U, Pluzanska A, Szczesna A, Kaukel E, Roubec J, De Rosa F, et al. Phase III study of erlotinib in combination with cisplatin and gemcitabine in advanced non-small-cell lung cancer: the Tarceva Lung Cancer Investigation Trial. J Clin Oncol. 2007;25:1545-52.

17. Lee DH, Lee JS, Kim SW, Rodrigues-Pereira J, Han B, Song XQ, et al. Three-arm randomised controlled phase 2 study comparing pemetrexed and erlotinib to either pemetrexed or erlotinib alone as second-line treatment for never-smokers with nonsquamous non-small cell lung cancer. Eur J Cancer. 2013;49: 3111-21.

18. Schemper M, Smith TL. A note on quantifying follow-up in studies of failure time. Control Clin Trials. 1996;17:343-6.

19. Mok TS, Wu YL, Thongprasert S, Yang CH, Chu DT, Saijo N, et al. Gefitinib or carboplatin-paclitaxel in pulmonary adenocarcinoma. N Engl J Med. 2009;361:947-57.

20. Von Pawel J, Papai-Szekely Z, Vinolas N, Sederholm C, Klima M, Desaiah D, et al. A randomized phase II study of pemetrexed versus pemetrexed plus erlotinib in second-line treatment for locally advanced or metastatic, nonsquamous NSCLC. J Clin Oncol. 2011;29(S):Abstr 7526.

21. Wu YL, Lee JS, Thongprasert S, Yu CJ, Zhang L, Ladrera G, et al. Intercalated combination of chemotherapy and erlotinib for patients with advanced stage non-small-cell lung cancer (FASTACT-2): a randomised, double-blind trial. Lancet Oncol. 2013;14:777-86.

22. Brugger W, Triller N, Blasinska-Morawiec M, Curescu S, Sakalauskas R, Manikhas GM, et al. Prospective molecular marker analyses of EGFR and KRAS from a randomized, placebo-controlled study of erlotinib maintenance therapy in advanced non-small-cell lung cancer. J Clin Oncol. 2011;29: 4113-20.

23. Gahr S, Stoehr R, Geissinger E, Ficker JH, Brueckl WM, Gschwendtner A, et al. EGFR mutational status in a large series of Caucasian European NSCLC patients: data from daily practice. Br J Cancer. 2013;109:1821-8.

24. Han JY, Park K, Kim SW, Lee DH, Kim HY, Kim HT, et al. First-SIGNAL: first-line single-agent iressa versus gemcitabine and cisplatin trial in never-smokers with adenocarcinoma of the lung. J Clin Oncol. 2012;30:1122-8. 


\section{Appendix}

A

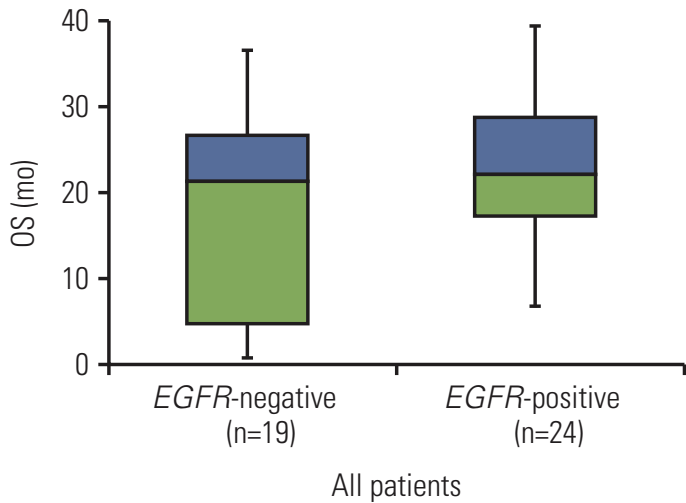

C
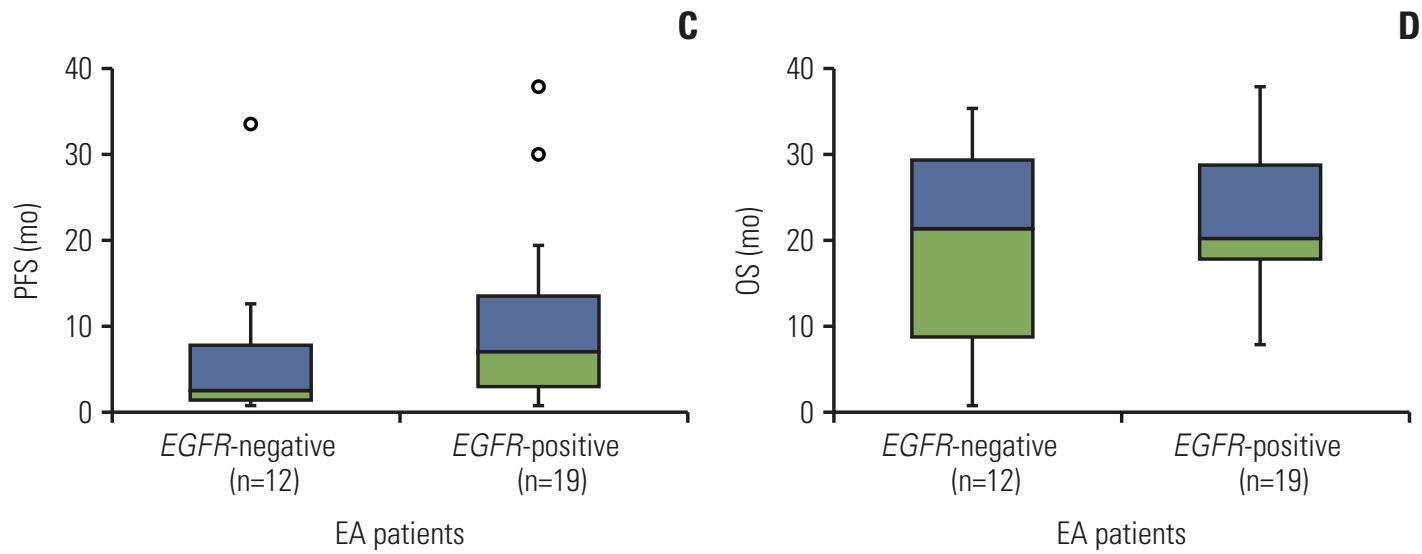

Appendix 1. Box and scatter plots of progression-free survival (PFS) and overall survival (OS) for epidermal growth factor receptor (EGFR) mutation positive and EGFR mutation-negative subsets. (A, B) Box plot of PFS and OS, respectively, for the entire population. (C, D) Box plot of PFS and OS, respectively, for East Asian (EA) population. (Continued to the next page) 
E
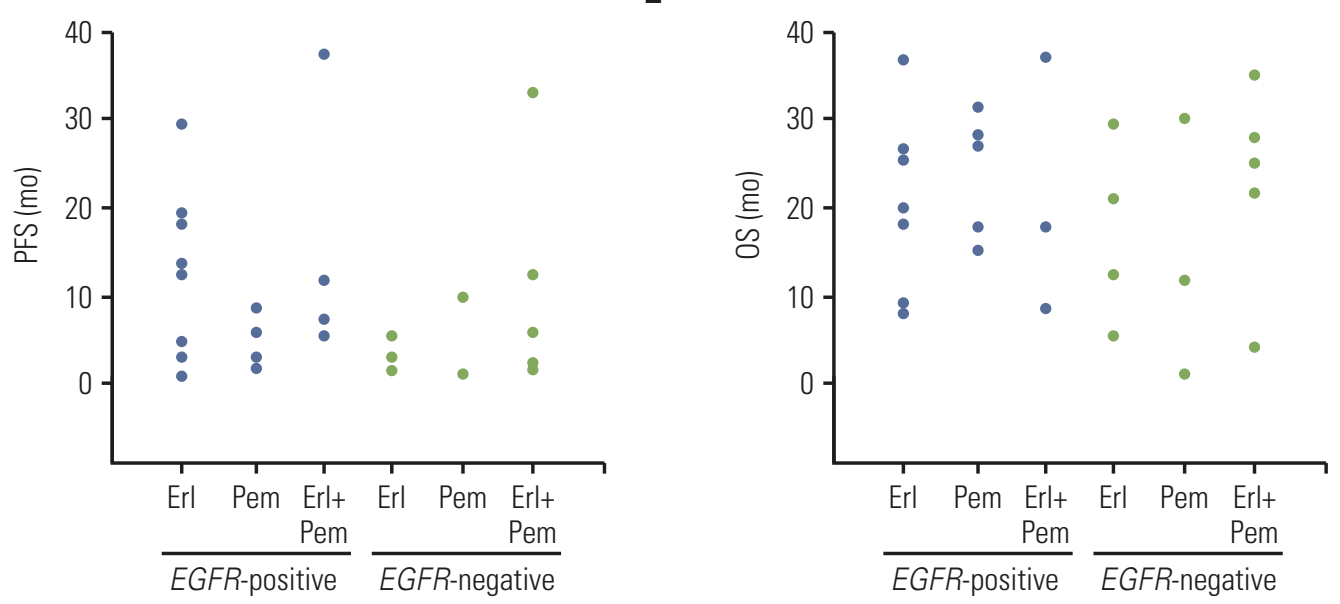

G

H
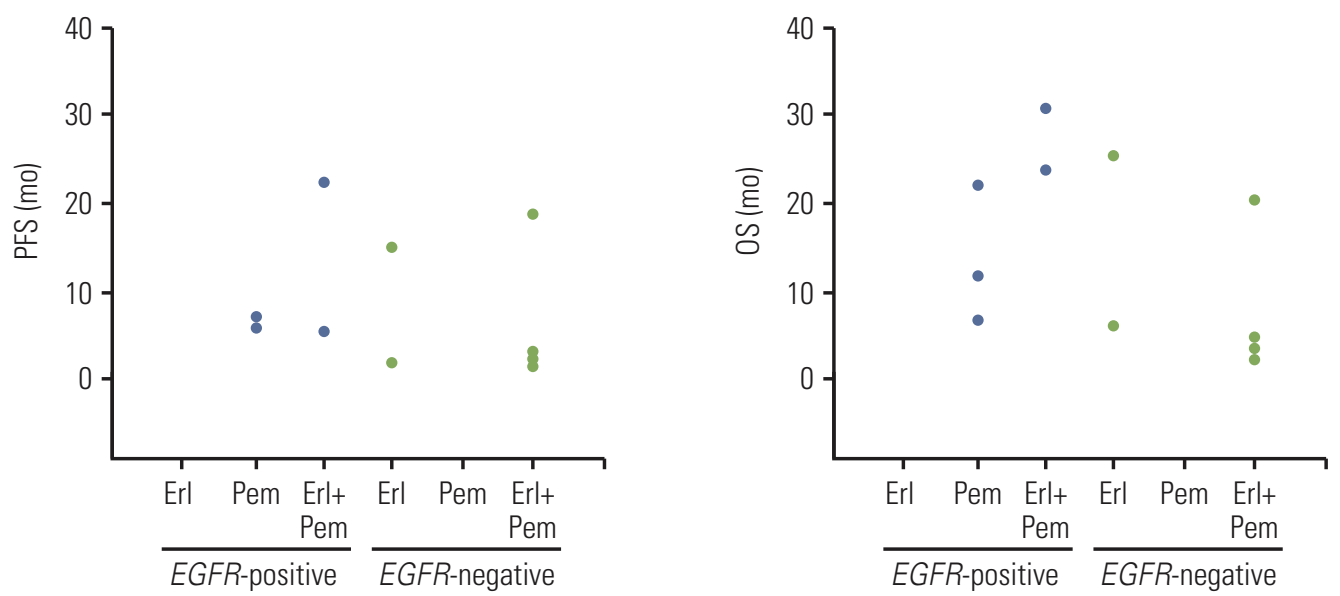

Appendix 1. (Continued from the previous page) (E, F) Scatter plot of PFS and OS, respectively, by treatment group in the EA population. (G, H) Scatter plot of PFS and OS, respectively, by treatment group in the non-EA population. Note: Patient with any EGFR mutation detected was categorized as mutated (positive); patient without EGFR mutations detected was categorized as non-mutated (negative); (A-D) the distribution of the middle half of the data was displayed by a box with the bottom, center, and top lines indicating quartile 1, median, and quartile 3, respectively. Potential outliers are plotted as individual points. The vertical lines extend from the bottom and top of the box to the smallest or largest non-outliers. $(G, H)$ There were no non-EA erlotinib-treated EGFR mutation positive patients or non-EA pemetrexed-treated EGFR mutation-negative patients. Erl, erlotinib; Pem, pemetrexed. 